Article

\title{
An Analog Circuit Fault Diagnosis Method Based on Circle Model and Extreme Learning Machine
}

\author{
Sumin Guo ${ }^{1}$, Bo Wu ${ }^{2, *}$, Jingyu Zhou ${ }^{2}$, Hongyu Li ${ }^{1, *}$, Chunjian $\mathrm{Su}^{3}$, Yibo Yuan ${ }^{1}$ and Kebao Xu ${ }^{1}$ \\ 1 College of Ocean Science and Engineering, Shandong University of Science and Technology, \\ Qingdao 266590, China; skd994055@sdust.edu.cn (S.G.); luckyyuanyibo@163.com (Y.Y.); \\ skdxkb@163.com (K.X.) \\ 2 China Electronics Technology Group Corporation No.41 Research Institute, Qingdao 266555, China; \\ blueangel19841227@163.com \\ 3 College of Mechanical and Electronic Engineering, Shandong University of Science and Technology, \\ Qingdao 266590, China; suchunjian2008@163.com \\ * Correspondence: wu28429361@163.com (B.W.); skdlhy@163.com (H.L.)
}

Received: 20 January 2020; Accepted: 27 March 2020; Published: 31 March 2020

\begin{abstract}
The fault diagnosis of analog circuits faces problems, such as inefficient feature extraction and fault identification. To solve the problems, this paper combines the circle model and the extreme learning machine (ELM) into a fault diagnosis method for the linear analog circuit. Firstly, a circle model for the voltage features of fault elements was established in the complex domain, according to the relationship between the circuit response, element position and circuit topology. To eliminate the impacts of tolerances and signal aliasing, the 3D feature was introduced to make the indistinguishable features in fuzzy groups distinguishable. Fault feature separability is very important to improve the fault diagnosis accuracy. In addition, an effective classier can improve the precision and the time taken. With less computational complexity and a simpler process, the ELM algorithm has a fast speed and a good classification performance. The effectiveness of the proposed method is verified by simulation. The simulation results show the ELM-based algorithm classifier with the circle model can enhance precision and reduce time taken by about $80 \%$ in comparison with other methods for analog circuit fault diagnosis. To sum up, this proposed method offers a fault diagnosis method that reduces the complexity in generating fault features, improves the isolation probability of faults, speeds up fault classification, and simplifies fault testing.
\end{abstract}

Keywords: analog circuit; feature engineering; fault diagnosis; circle model; extreme learning machine (ELM)

\section{Introduction}

Since the 1970s, analog circuit fault diagnosis has become a research hotspot in the field of electronic testing, and gradually formed a relatively complete theoretical system [1]. In order to ensure the test quality of circuits under test (CUT) at a low cost, many fault models and methods have been proposed [2-24]. One of the most widely used is the fault dictionary method [6]. The fault dictionary method is based on the simulation of circuit faults and the mapping relationship between fault features and fault components to form a fault dictionary. But it also has the following shortcomings: it can only diagnose catastrophic faults (hard faults) and discrete parameter fault types and the fault dictionary increases with the increase of components. Parameter fault diagnosis (also known as soft fault) is still an Non-deterministic (NP)-hard problem. With the development of modern information processing technology and artificial intelligence, starting from 1990's, a new generation of the analog circuit fault diagnosis method represented by machine learning algorithms has been widely developed. Modern 
analog circuit fault diagnosis methods can be divided into two main categories: model-based fault diagnosis methods and classifier based fault diagnosis methods.

The model-based fault diagnosis methods include the discrete parameter model, slope fault diagnosis, symbolic analysis, and so on. However, the discrete parameter model cannot characterize the fault completely, for the input/output $(\mathrm{I} / \mathrm{O})$ signals and element parameters all change continuously. The slope fault diagnosis only applies to linear circuits [3,4]. The fault of an element can be modeled with the slope as the fault feature. Nonetheless, slope fault diagnosis only works when the measuring points outnumber the faults to be modeled. The applicable scope of symbolic analysis is limited by its reliance on the transfer function [5]. Based on complex domain modeling, Tian et al. [6] and Yang et al. [9] proposed a method that creates a unified fault model called the circle model for all parameters, using only a few measuring points. Due to the presence of tolerances, this method cannot diagnose the critical regions of different circuit states accurately, which suppresses the test accuracy.

The classifier-based fault diagnosis methods are generally implemented in three steps: collecting features of the circuit in different states, training the collected features with a classifier, and diagnosing the fault based on the training data. These methods typically adopt classifiers like support vector machine (SVM) [7], least squares support vector machine (LS-SVM) [8], extreme learning machine (ELM) $[15,20]$, and so on. The SVM or LS-SVM algorithm is used to map the lower-dimensional nonlinear response space into the higher-dimensional feature space for effective classification. However, this algorithm has a much higher time cost because of complex computation of SVM, processes of test signal generator and test structure, and has unstable testing accuracy because of reduced precision in case of compressing the sampled space. The computational complexity of ELM algorithm in sample classification is much lower than that of SVM. Furthermore, trade-off parameters are not sensitive to the accuracy of ELM classification, so ELM has a good classification performance without optimization of trade-off parameters in case of compressing sampled space.

For modern intelligent diagnosis of analog circuit faults, the key lies in the extraction of fault features and the design of classification algorithms. In fact, the calculation rate and diagnosis efficiency of the diagnosis system directly hinge on the excellence of the fault feature extraction methods, especially those capable of extracting the features that clearly distinguishes between different types of faults.

During the failure of the analog circuit, the response output collected at the output point is often nonstationary, nonlinear, and time-varying. The original signals contain redundant information and have high dimensionality. If directly applied in fault diagnosis, these signals will push up the computing load and drag down the diagnosis efficiency and accuracy. To clearly distinguish between fault modes, it is critical to effectively process fault signals by the appropriate fault feature extraction method.

Through the above analysis, this paper decides to develop a novel fault diagnosis method for analog circuits, drawing on the model-based and classifier-based fault diagnosis methods. The circle modeling method is a model-based fault diagnosis method and it can characterize all possible faults in an easy way for both soft faults and hard faults. And the ELM classification algorithm is not time consuming as a classifier. The circle model is used for feature extraction and the ELM classification algorithm is used as a fault classifier. The proposed method of circle model-based feature extraction and the ELM classification algorithm combination will fully inherit the completeness of the circle model and the fast classification of the ELM, and achieve higher accurate and efficient fault diagnosis of analog circuit.

\section{Feature Engineering Based on Circle Model}

\subsection{Principle of Circle Modeling}

Figure 1 illustrates the procedure of circle modeling. In Figure $1 \mathrm{a}, \mathrm{N}$ is a linear time-invariant circuit, $\dot{U}_{\mathrm{s}}$ is the voltage phasor of the independent voltage source that stimulates $\mathrm{N}, \dot{U}_{\mathrm{o}}$ is the output 
voltage phasor, $x$ is a passive element, and $\dot{U}_{\mathrm{x}}$ is the voltage phasor across $x$. The theory is described below $[6,9,10]$ :

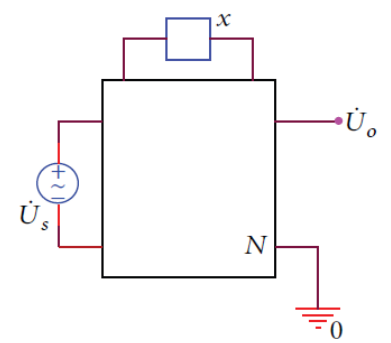

(a) Original circuit

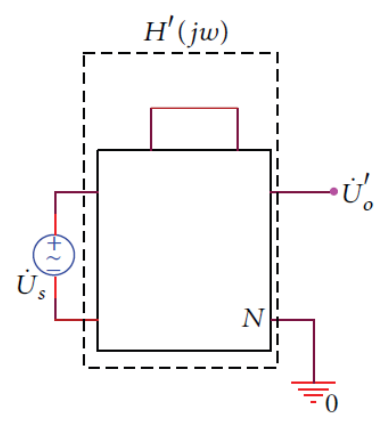

(c) N stimulated by $\dot{\mathrm{U}}_{s}$

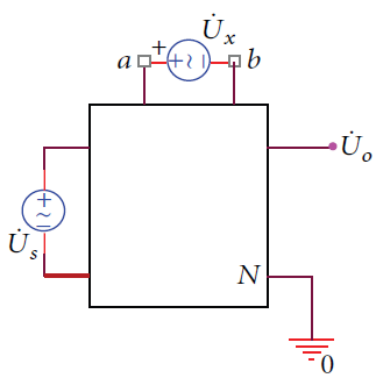

(b) Equivalent circuit

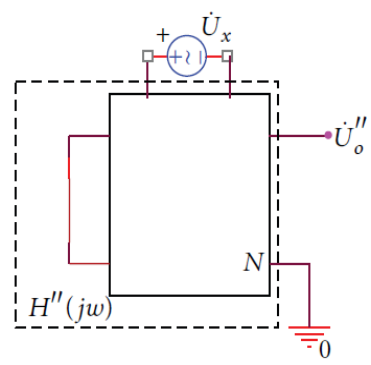

(d) N stimulated by $\dot{\mathrm{U}}_{x}$

Figure 1. The procedure of circle modeling.

According to the substitution theorem, the passive element $x$ can be replaced with an independent voltage source with voltage phasor $\dot{U}_{\mathrm{x}}$ (Figure 1b). The voltage phasor $\dot{U}_{\mathrm{x}}$ can be derived by the Thévenin's theorem:

$$
\dot{U}_{x}=\dot{U}_{o c} \frac{z_{x}}{z_{\mathrm{o}}+z_{x}}
$$

where, $\dot{U}_{\mathrm{oc}}$ is the open circuit voltage phasor across terminals a and $\mathrm{b}$ in the equivalent circuit (Figure $1 b$ ); $Z_{\mathrm{o}}$ is the Thévenin impedance between terminals $a$ and $b$. According to the Thévenin's theorem, $\dot{U}_{\mathrm{oc}}$ and $Z_{\mathrm{o}}$ are independent from the value of $\mathrm{x}$ and uniquely determined by its location and the fault-free elements in $\mathrm{N}$.

According to the substitution theorem, the output voltage phasor $\dot{U}_{\mathrm{o}}$ in Figure 1a is equal to $\dot{U}_{\mathrm{o}}$ in Figure $1 \mathrm{~b}$, where $\mathrm{N}$ is stimulated by two independent voltage sources $\dot{U}_{\mathrm{s}}$ and $\dot{U}_{\mathrm{x}}$.

According to the superposition theorem, the $\dot{U}_{\mathrm{o}}$ in Figure $1 \mathrm{~b}$ equals the algebraic sum of the responses to $\dot{U}_{\mathrm{s}}$ or $\dot{U}_{\mathrm{x}}$. Figure 1c,d illustrate the circuit under the operation of $\dot{U}_{\mathrm{s}}$ and that of $\dot{U}_{\mathrm{x}}$ respectively. The following equation holds under the operation of $\dot{U}_{\mathrm{s}}$ :

$$
\dot{U}_{o}^{\prime}=\dot{U}_{s} H^{\prime}(j \omega)
$$

where, $H^{\prime}(j \omega)$ is independent from the value of $x$.

The following equation holds under the operation of $\dot{U}_{\mathrm{x}}$ :

$$
\dot{U_{o}^{\prime \prime}}=\dot{U}_{x} H^{\prime \prime}(j \omega)
$$

where, $H^{\prime \prime}(j \omega)$ is independent from the value of $\mathrm{x}$. 
According to the superposition theorem, we have:

$$
\dot{U}_{o}=\dot{U}_{o}^{\prime}+\dot{U}_{o}^{\prime \prime}=\dot{U}_{s} H^{\prime}(j \omega)+\dot{U}_{x} H^{\prime \prime}(j \omega)
$$

Replacing $\dot{U}_{\mathrm{x}}$ with Formula (1), we have:

$$
\begin{gathered}
\dot{U}_{o}=\dot{U}_{\mathrm{s}} H^{\prime}(j \omega)+\dot{U}_{o c} \frac{z_{x}}{z_{0}+z_{x}} H^{\prime \prime}(j \omega) \\
\Rightarrow \frac{z_{o}}{z_{x}}=\frac{\dot{U}_{o c} H^{\prime \prime}(j \omega)-\left(\dot{U} o-\dot{U}_{s} H^{\prime}(j \omega)\right)}{\dot{U}_{o-} \dot{U}_{s} H^{\prime}(j \omega)} \\
=\frac{\dot{U}_{o c} H^{\prime \prime}(j \omega)}{\dot{U}_{o s}}-1
\end{gathered}
$$

where,

$$
\dot{U}_{o s}=\dot{U} o-\dot{U}_{s} H^{\prime}(j \omega)
$$

Without loss of generality, it is assumed that:

$$
\left\{\begin{array}{l}
Z_{o}=R_{o}+j X_{o} \\
\dot{U}_{o s}=U_{o s r}+j U_{o s j} \\
\dot{U}_{o c} H^{\prime \prime}(j \omega)=m+j n
\end{array}\right.
$$

where, $j$ is the imaginary unit of the imaginary part and denotes the imaginary part. Since $\dot{U}_{\mathrm{s}}, H^{\prime}(j \omega)$, $\dot{U}_{o c}, H^{\prime \prime}(j \omega)$, and $Z_{o}$ are independent from $Z_{x}, R_{0}, X_{0}, \mathrm{~m}$ and $\mathrm{n}$ must be independent from $Z_{x}$, too.

Then, Formula (6) can be rewritten as:

$$
\frac{R_{\mathrm{o}}+j X_{o}}{Z_{x}}=\frac{m U_{o s r}+n U_{o s j}}{U_{o s r}^{2}+U_{o s j}^{2}}-1+j \frac{n U_{o s r}-m U_{o s j}}{U_{o s r}^{2}+U_{o s j}^{2}}
$$

If fault component $x$ is a resistor and $Z_{x}=R_{x}$, the following equations hold:

$$
\left\{\begin{array}{l}
\frac{R_{0}}{R_{x}}=\frac{m U_{o s r}+n U_{o s j}}{U_{o s r}^{2}+U_{o s j}^{2}}-1 \\
\frac{X_{o}}{R_{x}}=\frac{n U_{o s r}-m U_{o s j}}{U_{o s r}^{2}+U_{o s j}^{2}}
\end{array}\right.
$$

Eliminating $R_{x}$, the following formula can be obtained:

$$
\begin{aligned}
& \frac{R_{\mathrm{o}}}{X_{o}} \frac{n U_{o s r}-m U_{o s j}}{U_{o s r}^{2}+U^{2}{ }_{o s j}}-\frac{m U_{o s r}+n U_{o s j}}{U_{o s r}^{2}+U^{2}{ }_{o s j}}+1=0 \\
\Rightarrow & \frac{R_{o}}{X_{o}}\left(n U_{o s r}-m U_{o s j}\right)-\left(m U_{o s r}+n U_{o s j}\right)+U_{o s r}^{2}+U_{o s j}^{2}=0 \\
\Rightarrow & U_{o s r}^{2}+\left(\frac{R_{o}}{X_{o}} \mathrm{n}-m\right) U_{o s r}+U_{o s r}^{2}-\left(\frac{R_{o}}{X_{o}} \mathrm{~m}+\mathrm{n}\right) U_{o s j}=0 \\
\Rightarrow & {\left[U_{o s r}-\frac{1}{2}\left(m-\frac{R_{o}}{X_{0}} \mathrm{n}\right)\right]^{2}+\left[U_{o s j}-\frac{1}{2}\left(n+\frac{R_{o}}{X_{o}} m\right)\right]^{2} } \\
= & \frac{1}{4}\left[\left(m-\frac{R_{o}}{X_{0}} n\right)^{2}+\left(n+\frac{R_{o}}{X_{0}} m\right)^{2}\right]
\end{aligned}
$$

In Formula (7), $\dot{U}_{o s}=\dot{U} o-\dot{U}_{s} H^{\prime}(j \omega)$. If $\dot{U}_{o}=U_{o r}+j U_{o j}$ and $\dot{U}_{s} H^{\prime}(j \omega)=U_{o r}^{\prime}+j U_{o j}^{\prime}$, the following equations can be obtained:

$$
\left\{\begin{array}{l}
U_{o s r}=U_{o r}-U_{o r}^{\prime} \\
U_{o s j}=U_{o j}-U_{o j}^{\prime}
\end{array}\right.
$$


Replacing $U_{o s r}$ and $U_{o s j}$ in Formula (12) with Formula (13), we have:

$$
\begin{gathered}
{\left[\mathrm{U}_{\mathrm{or}}-U_{o r}^{\prime}-\frac{1}{2}\left(m-\frac{R_{\mathrm{o}}}{\mathrm{X}_{o}} n\right)\right]^{2}+\left[U_{o j}-U_{o j}^{\prime}-\frac{1}{2}\left(n+\frac{R_{o}}{X_{o}} m\right)\right]^{2}} \\
=\frac{1}{4}\left[\left(m-\frac{R_{o}}{X_{o}} n\right)^{2}+\left(n+\frac{R_{o}}{X_{o}} m\right)^{2}\right] \\
\Rightarrow\left(\mathrm{U}_{\mathrm{o} r}-a\right)^{2}+\left(U_{\mathrm{oj}}-b\right)^{2}=r^{2}
\end{gathered}
$$

where,

$$
\left\{\begin{array}{l}
\mathrm{a}=U_{0 r}^{\prime}+\frac{1}{2}\left(m-\frac{R_{0}}{\mathrm{X}_{0}} n\right), \\
b=U_{0 j}^{\prime}+\frac{1}{2}\left(n+\frac{R_{0}}{X_{0}} m\right), \\
r=\frac{1}{2} \sqrt{\left(m-\frac{R_{0}}{X_{0}} n\right)^{2}+\left(n+\frac{R_{0}}{X_{0}} m\right)^{2}}
\end{array}\right.
$$

Obviously, Formula (15) is a circle equation, with a and b being the $U_{o r}$ - and $U_{o j}$-coordinates of the center and $\mathrm{r}$ being the radius. Because $R_{0}, X_{0}, m$, and $n$ are independent from the value of $x$, a and $b$ must be independent from the value of $x$, too. Therefore, Formula (15) always holds regardless of the fault type occurring to $x$. Hence, the circle equation governing the relationship between $U_{o r}$ and $U_{o j}$ is applicable to both hard and soft faults, and thus selected as the circuit fault model of this research. The parameters $a, b$, and $r$ that govern the circle equation are characteristic parameters. If $x$ is a dynamic element, the same conclusions can be reached. In addition, because Formula (15) is independent from the value of $x$ to be modeled. The fault-free output voltage, $\dot{U}_{o}=U_{o r}+j U_{o j}$ must satisfy (15). Therefore, all characteristic curves pass through the fault-free point $\left(U_{o r}, U_{o j}\right)[13]$.

\subsection{Circle Model-Based Feature Engineering}

Circle modeling was adopted to collect the necessary features for the classifier. Take the circuit of a Leapfrog filter as an example, with a $5 \mathrm{~V}, 1 \mathrm{kHz}$ sine wave as the excitation signal by using MATLAB calling PSPICE. The settings of circuit parameters are shown in Figure 2. The circuit features are collected at the second-stage filtered output point (out).

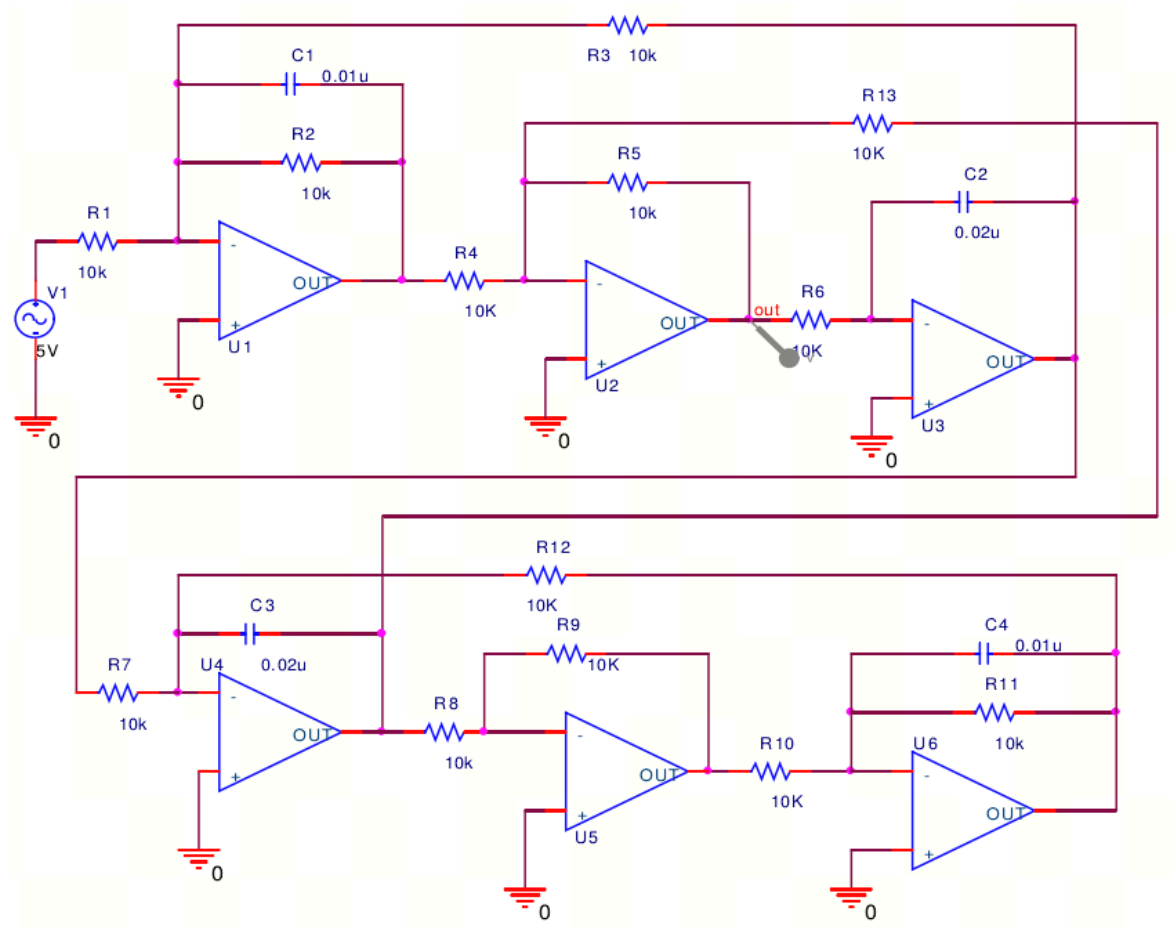

Figure 2. Diagram of Leapfrog circuit. 


\subsubsection{Circle Model-Based Feature Extraction}

According to the circle model theory, the real and imaginary parts of the fault voltage of a linear circuit can be described by the circle equation on the complex field, and all pass through the fault-free point. Thus, a circle can be defined by three points and only three simulations are needed to obtain the fault features of the analog element in all fault states.

If a component suffers from a catastrophic failure (e.g., open circuit and short circuit), the circle model of the element fault can be established based on the output voltages of the element under open circuit and short circuit and the fault-free point that the fault voltages of all element pass through. Suppose the parameter scanning range is $X / 10^{4} \leq p_{x} \leq 10^{4} X$ ( $X$ is the parameter value of an element). Taking a resistor for instance, the resistance is $100 \mathrm{M} \Omega$ in open circuit and $1 \Omega$ in short circuit.

Here, the fault parameters of each element are scanned within the preset range, revealing the output voltage of each element. Then, the analog voltage phasor $\dot{U}_{o}$ was decomposed into a real part $U_{o r}$ and an imaginary part $U_{o j}$. Three voltages $\left\{\dot{U}_{1}, \dot{U}_{2}, \dot{U}_{o}\right\}$ respectively denoting short circuit, open circuit and free fault can be obtained through one parametric sweep simulation. Then the following equations are obtained:

$$
\left\{\begin{array}{l}
\left(U_{o r}-a\right)^{2}+\left(U_{o j}-b\right)^{2}=r^{2} \\
\left(U_{1 r}-a\right)^{2}+\left(U_{1 j}-b\right)^{2}=r^{2} \\
\left(U_{2 r}-a\right)^{2}+\left(U_{2 j}-b\right)^{2}=r^{2}
\end{array}\right.
$$

The output voltage of the test point of the free fault can be expressed as $(2.394,3.175)$ in the complex field. To the fault model, only two more points need to be measured for each component. The simulation results and the parameters of the fault model are shown in Table 1.

Table 1. Fault model parameter of the Leapfrog circuit.

\begin{tabular}{|c|c|c|c|c|}
\hline \multirow{2}{*}{ Fault Source } & \multirow{2}{*}{ Sampled Value } & \multirow{2}{*}{ Output Voltage } & \multicolumn{2}{|c|}{ Model Parameter } \\
\hline & & & Circle Center $(a, b)$ & Radius $\mathbf{r}$ \\
\hline \multirow{2}{*}{ R1 } & $1 \Omega$ & $2.383 \times 10^{4}, 3.158 \times 10^{4}$ & \multirow{2}{*}{-} & \multirow{2}{*}{-} \\
\hline & $100 \mathrm{M} \Omega$ & $2.394 \times 10^{-2}, 3.176 \times 10^{-2}$ & & \\
\hline \multirow{2}{*}{ R2 } & $1 \Omega$ & $5.080 \times 10^{-4}, 5.548 \times 10^{-4}$ & \multirow{2}{*}{$(-15.393,14.093)$} & \multirow{2}{*}{20.870} \\
\hline & $100 \mathrm{M} \Omega$ & $4.261,7.073$ & & \\
\hline \multirow{2}{*}{ R3 } & $1 \Omega$ & $6.061 \times 10^{-8}, 6.283 \times 10^{-4}$ & \multirow{2}{*}{$(3.3031,-0.0005)$} & \multirow{2}{*}{3.303} \\
\hline & $100 \mathrm{M} \Omega$ & $6.108,1.744$ & & \\
\hline \multirow{2}{*}{ R4 } & $1 \Omega$ & $6.104 \times 10^{-4}, 6.283$ & \multirow{2}{*}{$(-0.8641,3.1416)$} & \multirow{2}{*}{3.258} \\
\hline & $100 \mathrm{M} \Omega$ & $6.114 \times 10^{-2}, 1.746 \times 10^{-2}$ & & \\
\hline \multirow{2}{*}{ R5 } & $1 \Omega$ & $3.585 \times 10^{-4},-2.25 \times 10^{-4}$ & \multirow{2}{*}{$(1.0614,1.6897)$} & \multirow{2}{*}{1.995} \\
\hline & $100 \mathrm{M} \Omega$ & $-4.321 \times 10^{-1}, 3.013$ & & \\
\hline \multirow{2}{*}{ R6 } & $1 \Omega$ & $-4.225 \times 10^{-5}, 3.000 \times 10^{-4}$ & \multirow{2}{*}{$(2.758,0.4105)$} & \multirow{2}{*}{2.788} \\
\hline & $100 \mathrm{M} \Omega$ & $3.642,-2.234$ & & \\
\hline \multirow{2}{*}{ R7 } & $1 \Omega$ & $-1.573 \times 10^{-4}, 5.557 \times 10^{-4}$ & \multirow{2}{*}{$(2.3839,0.6925)$} & \multirow{2}{*}{2.483} \\
\hline & $100 \mathrm{M} \Omega$ & $4.861,8.568 \times 10^{-1}$ & & \\
\hline \multirow{2}{*}{ R8 } & $1 \Omega$ & $4.863,8.145 \times 10^{-1}$ & \multirow{2}{*}{$(5.1950,3.6332)$} & \multirow{2}{*}{2.838} \\
\hline & $100 \mathrm{M} \Omega$ & $3.988,6.202$ & & \\
\hline \multirow{2}{*}{ R9 } & $1 \Omega$ & $4.038,6.224$ & \multirow{2}{*}{$(5.1950,3.6327)$} & \multirow{2}{*}{2.838} \\
\hline & $100 \mathrm{M} \Omega$ & $4.810,8.212 \times 10^{-1}$ & & \\
\hline \multirow{2}{*}{ R10 } & $1 \Omega$ & $-0.6481,3.870$ & \multirow{2}{*}{$(5.1950,3.6332)$} & \multirow{2}{*}{2.838} \\
\hline & $100 \mathrm{M} \Omega$ & $4.863,8.145 \times 10^{-1}$ & & \\
\hline
\end{tabular}


Table 1. Cont.

\begin{tabular}{|c|c|c|c|c|}
\hline \multirow{2}{*}{ Fault Source } & \multirow{2}{*}{ Sampled Value } & \multirow{2}{*}{ Output Voltage } & \multicolumn{2}{|c|}{ Model Parameter } \\
\hline & & & Circle Center $(a, b)$ & Radius $\mathbf{r}$ \\
\hline \multirow{2}{*}{ R11 } & $1 \Omega$ & $4.038,6.224$ & \multirow{2}{*}{$(19.153,-3.8939)$} & \multirow{2}{*}{18.189} \\
\hline & $100 \mathrm{M} \Omega$ & $1.298,-4.253 \times 10^{-1}$ & & \\
\hline \multirow{2}{*}{ R12 } & $1 \Omega$ & $-0.6481,3.870$ & \multirow{2}{*}{$(5.1950,3.6332)$} & \multirow{2}{*}{2.838} \\
\hline & $100 \mathrm{M} \Omega$ & $4.863,8.145 \times 10^{-1}$ & & \\
\hline \multirow{2}{*}{ R13 } & $1 \Omega$ & $-1.602 \times 10^{-4}, 5.512 \times 10^{-4}$ & \multirow{2}{*}{$(2.3839,0.6925)$} & \multirow{2}{*}{2.482} \\
\hline & $100 \mathrm{M} \Omega$ & $4.861,8.568 \times 10^{-1}$ & & \\
\hline \multirow{2}{*}{$\mathrm{C} 1$} & $1 \mathrm{pF}$ & $1.210,3.466$ & \multirow{2}{*}{$(1.3481,1.4736)$} & \multirow{2}{*}{1.997} \\
\hline & $100 \mathrm{mF}$ & $8.835 \times 10^{-4},-8.085 \times 10^{-4}$ & & \\
\hline \multirow{2}{*}{$\mathrm{C} 2$} & $1 \mathrm{pF}$ & $-1.993 \times 10^{-5}, 1.502 \times 10^{-4}$ & \multirow{2}{*}{$(2.758,0.4105)$} & \multirow{2}{*}{2.788} \\
\hline & $100 \mathrm{mF}$ & $3.585,-2.252$ & & \\
\hline \multirow{2}{*}{$\mathrm{C} 3$} & $1 \mathrm{pF}$ & $2.262,7.341 \times 10^{-1}$ & \multirow{2}{*}{$(3.5283,1.8896)$} & \multirow{2}{*}{1.714} \\
\hline & $100 \mathrm{mF}$ & $4.864,8.150 \times 10^{-1}$ & & \\
\hline \multirow{2}{*}{$\mathrm{C} 4$} & $1 \mathrm{pF}$ & $3.452,3.081$ & \multirow{2}{*}{$(3.0696,4.7786)$} & \multirow{2}{*}{1.740} \\
\hline & $100 \mathrm{mF}$ & $4.037,6.225$ & & \\
\hline
\end{tabular}

The three points of the measured data are taken to determine the characteristics of the analog circuit curves in Figure 3. It should be pointed out that because the parameter value of an actual analog component cannot be negative, the characteristic curve may not be a whole circle but an arc.

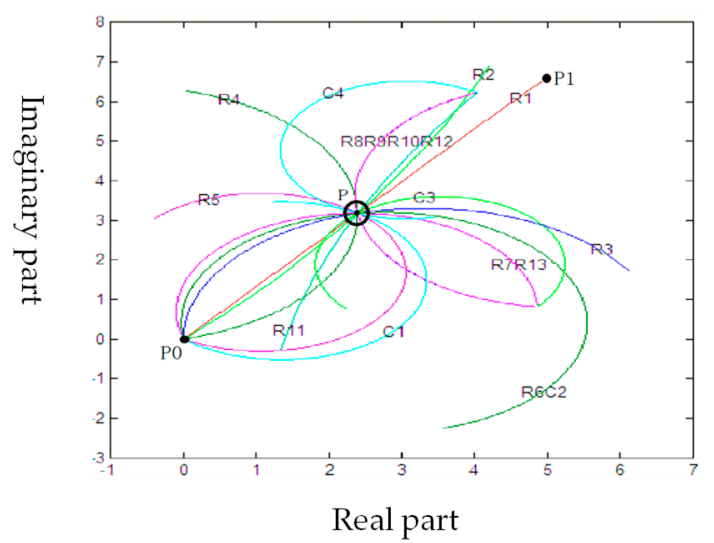

Figure 3. The circle model of Leapfrog circuit.

It can be seen from the Table 1 and Figure 3 that the component set $\{\mathrm{R} 6, \mathrm{C} 2\}$ has the same failure model $(2.758,0.4105) 2.788$, so they can't be distinguished and form a fuzzy group. Similarly, $\{R 7, R 13\}$ and $\{R 8, R 9, R 10, R 12\}$ are also fuzzy groups. The result of grouping is shown in Table 2. Except for the components of fuzzy group, other components have unique models. 
Table 2. Fuzzy groups of the Leapfrog circuit.

\begin{tabular}{cc}
\hline Fuzzy Groups & Fault Elements \\
\hline 1 & R1 \\
\hline 2 & R2 \\
\hline 3 & R3 \\
\hline 4 & R4 \\
\hline 5 & R5 \\
\hline 6 & R6, C2 \\
\hline 7 & R7, R13 \\
\hline 8 & R8, R9, R10, R12 \\
\hline 9 & R11 \\
\hline 10 & $\mathrm{C} 1$ \\
\hline 11 & $\mathrm{C} 3$ \\
\hline 12 & $\mathrm{C} 4$ \\
\hline
\end{tabular}

In the actual test, each element has a certain tolerance, and thus faces a deviation in the actual trajectory of the output voltage. To minimize the deviation, a classifier was introduced to diagnose and position the fault more accurately, in association with the simulated voltage features of the circle model in the complex domain.

\subsubsection{Feature Construction}

Using the circle model, it is easy to acquire the characteristic fault voltage curves of the simulated elements quickly. Under the presence of tolerances, however, some elements belong to fuzzy groups, due to the similarity between their characteristic curves. The elements in the same fuzzy group are indistinguishable. Direct positioning of such elements will lead to misclassification, reducing the accuracy of diagnostic positioning. The classification accuracy will also be suppressed, if the selected features for classification are not distinguishable. For example, points X1, X2 and X3 (Figure 4) are too close to each other at a certain frequency. These points and their tolerance areas will greatly affect the classification accuracy. Therefore, the fuzzy groups must be handled effectively before diagnosing circuit faults $[17,18]$.

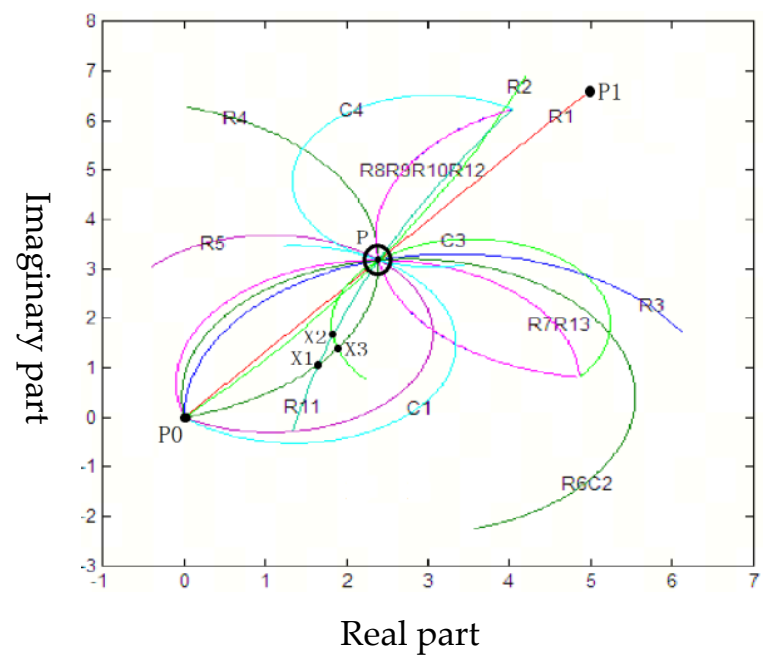

Figure 4. Sketch map of fuzzy groups. 
Considering the indistinguishability between 2D voltage features in the complex domain, this paper puts forward a 3D voltage feature construction method. First, all the points that are indistinguishable in 2D and distinguishable in 3D were identified based on the circle model of the original frequency. Then, the frequency was adjusted to find the second frequency making the originally indistinguishable voltage points distinguishable. The second frequency was inputted into the signal to compute the second set of complex domain features. In this way, a new feature was constructed as $\left(x_{f_{1}}, y_{f_{1}}, x_{f_{2}}, y_{f_{2}}\right)$, which can be compressed into a 3D feature $\left(x_{f_{1}}, \mathrm{y}_{f_{1}}, x_{f_{2}}\right)$.

As shown in Figure 5, the 3D map with frequency feature helps to differentiate between the voltage output points that are indistinguishable in 2D complex domain, in the light of their variation with frequencies in $3 \mathrm{D}$ space.

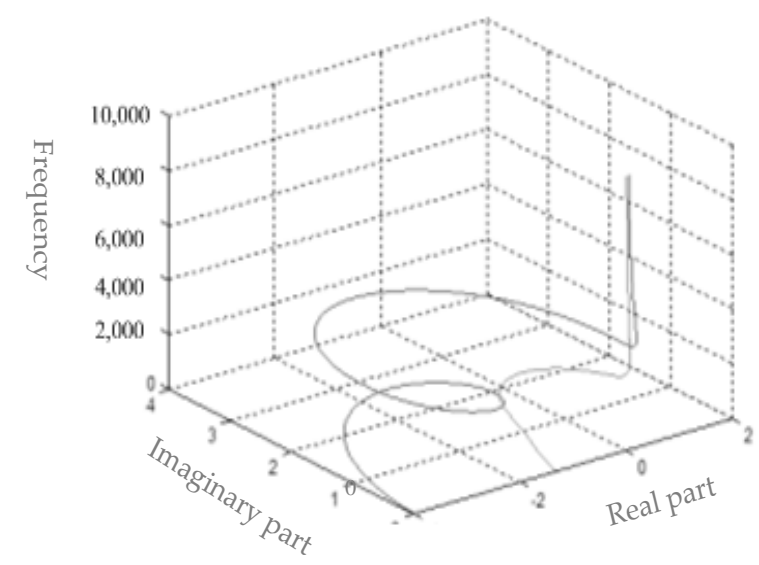

Figure 5. The 3D map of complex domain with frequency feature.

\subsubsection{Feature Preprocessing}

The normalization of features can improve the test accuracy of the classification algorithm. Here, a feature normalization method is developed based on the circle model. Take the circle model in Figure 3 for example. Since the arc trajectory for the fault voltage of each element must pass through the fault-free point $\mathrm{P}$, the theoretical fault-free point $\mathrm{P}$ based on voltage features was selected as the new origin of the complex domain in the translation operation. The setting of the new origin is reasonable, because all types of faults of Linear Time-Invariant systems (LTIs) change around point P.

Let $\dot{U}_{t}=x_{t}+j y_{t}$ be an output characteristic voltage of the circuit. Then, the current voltage of the fault-free point $\mathrm{P}$ is $\dot{U}_{0}=x_{0}+j y_{0}$, and the characteristic voltage after translation is $\left(x_{t}-x_{0}, y_{t}-y_{0}\right)$.

As shown in Figure 6, the characteristic voltages of different states after translation were distributed evenly around the fault-free point, which facilitates the compression of the eigenvalues to $[-1,1]$ and the application of the classifier. 


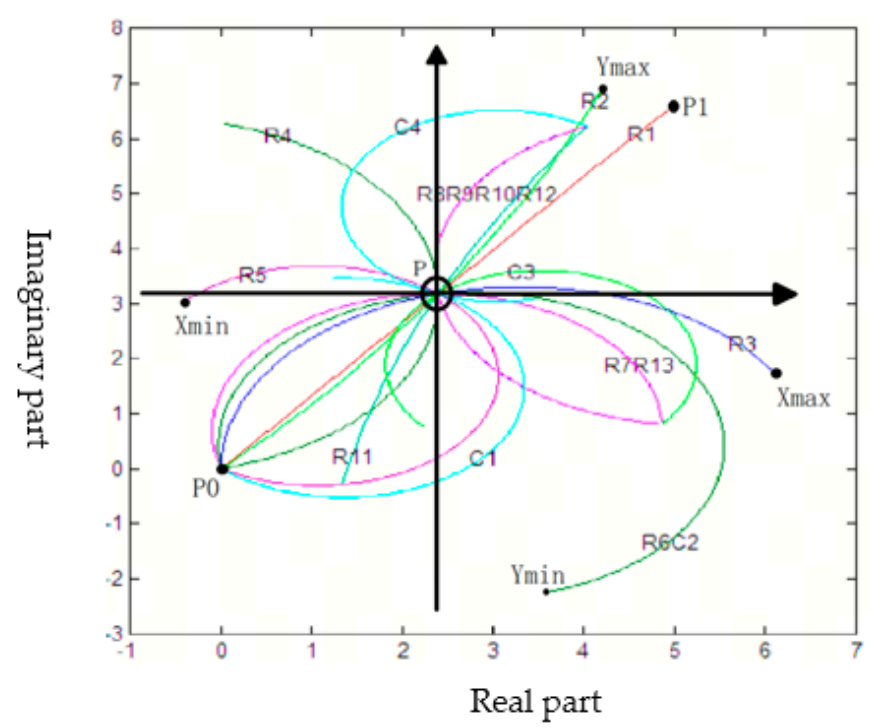

Figure 6. Feature normalization based on the circle model.

After the translation, the translated eigenvalues were compressed by a ratio equal to the difference between the maximum and minimum of the voltage features in the new coordinate system. Considering the effect of tolerances, the feature normalization formula can be expressed as:

$$
\left\{\begin{array}{l}
x=\frac{x_{t}-x_{0}}{\left(x_{\max }-x_{\min }\right)(1+\text { tor } \%)} \\
y=\frac{\left.y_{t}-y_{0}\right)}{\left(y_{\max }-y_{\min }\right)(1+\text { tor } \%)}
\end{array}\right.
$$

where, tor $\%$ is the tolerance parameter; $x_{t}$ and $y_{t}$ are the real and imaginary values of the translated circuit. The maximum and minimum of the voltage features were identified quickly by drawing a circle from three points. Then, the compression ratio was determined as the difference between the maximum and the minimum. On this basis, the voltage features were normalized by Formula (18), completing the feature preprocessing.

\subsection{The ELM Theory}

Proposed in 2006, the ELM is a learning method based on a feedforward neural network with a single hidden layer [19-22]. The theory of the ELM is briefly introduced below:

Let $\left(x_{i}, t_{i}\right)$ be $N$ different random samples, where $x_{i}=\left[x_{i 1}, x_{i 2}, \cdots, x_{i n}\right]^{T} \in R^{n}$ and $t_{i}=\left[t_{i 1}, t_{i 2}, \cdots, t_{i \mathrm{~m}}\right]^{T} \in R^{m}, N$ are the number of nodes in the hidden layer, and $g(x)$ is the activation function. Then, a neural network with a single hidden layer can be described as:

$$
\sum_{\mathrm{i}=1}^{\mathrm{N}} \beta_{\mathrm{i}} g_{i}\left(x_{j}\right)=\sum_{\mathrm{i}=1}^{\mathrm{N}} \beta_{\mathrm{i}} g_{i}\left(\mathrm{w}_{i} \cdot x_{j}+b_{i}\right)=o_{j}, j=1,2, \ldots, N
$$

where, $w_{i}=\left[w_{i 1}, w_{i 2}, \ldots, w_{i n},\right]^{T}$ and $\beta_{i}=\left[\beta_{i 1}, \beta_{i 2}, \ldots, \beta_{i \mathrm{~m}}\right]^{T}$ are the weight vector and weight between the $\mathrm{i}$-th hidden layer node and each input layer node, respectively; $b_{i}$ is the threshold of the i-th hidden layer node. The output of the ELM can be expressed as:

$$
f(x)=\sum_{i=1}^{L} \beta_{i} g\left(a_{i}, b_{i}, x\right)=\beta \cdot h(x)
$$


According Huang's Theory [16], the decision function of ELM can be expressed as:

$$
f(x)=\operatorname{sign}\left(\sum_{i=1}^{L} \beta_{i} G\left(a_{i}, b_{i}, x\right)\right)=\operatorname{sign}(\beta \cdot h(x))
$$

In the ELM algorithm, the input layer parameters are randomly set up while the weight coefficients of the output layer are obtained by solving the Moore-Penrose generalized inverse of the hidden layer output matrix. Therefore, the algorithm runs much faster than the other neural network with a single hidden layer and the support vector machine (SVM). The ELM classification algorithm can improve the fault diagnosis of linear circuit, in both training speed and test speed.

\subsection{Process of the Proposed Method}

The parameter simulation of analog circuit is completed by PSPICE software and the output voltages are extracted from the output file by MATLAB. The process of the method is presented as follows:

Step 1: Build a circle model through three points;

Step 2: Determine whether the 3D feature construction has been completed. If not, go to the next step. If it is completed, go to step 4;

Step 3: Judge the indiscernibility of fuzzy group;

Step 4: Find out the second frequency and construct three-dimensional features by the complex voltage domain features of twice frequency;

Step 5: Normalization processing is carried out and the feature preprocessing is completed;

Step 6: Train ELM;

Step 7: Test the model and realize the fault diagnosis of an analog circuit.

\section{Test Verification}

\subsection{Simulation Test 1}

The Tow-Thomas filter (Figure 7) was selected for simulation test 1 . The tolerances of the elements fall within $[-10 \%, 10 \%]$. The fault states of elements fall outside the tolerance interval: $[10 \%, 50 \%] \mathrm{U}[-50 \%,-10 \%]$.

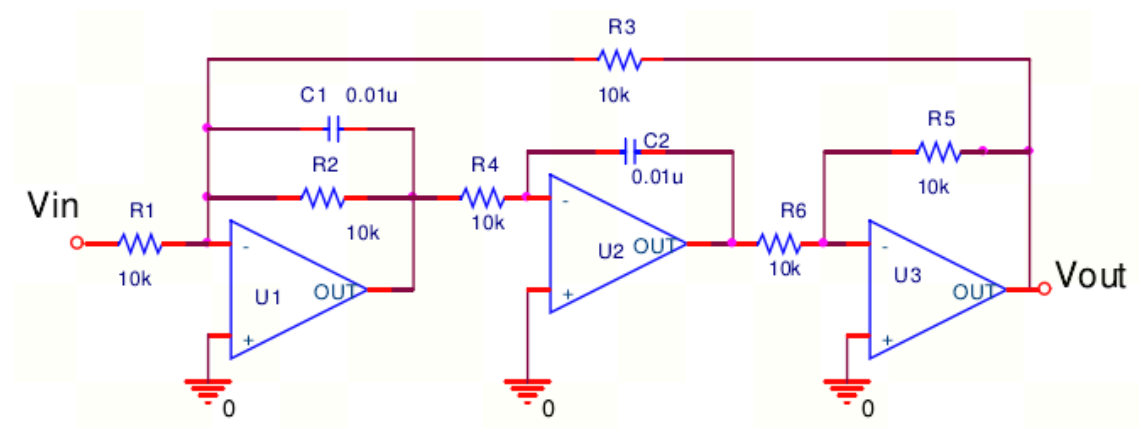

Figure 7. The Tow-Thomas filter.

The ELM is a classification algorithm based on the activation function. The classification effect of the algorithm depends on the test accuracy of the activation function relative to sample features. Hence, three popular activation functions, namely, Multiquadrics, Gaussian and Sigmoid, were selected for training and testing. The results of the three functions are recorded in Table 3 below. 
Table 3. The training and test results of different activation functions.

\begin{tabular}{ccc}
\hline Activation Function & Training Accuracy & Test Accuracy \\
\hline Multiquadrics & 92.4 & 91.2 \\
\hline Gaussian & 94.7 & 93.8 \\
\hline Sigmoid & 93.9 & 92.6 \\
\hline
\end{tabular}

As shown in Table 3, the Gaussian function achieved the highest training and test accuracies in fault diagnosis. Therefore, the Gaussian function was adopted as the activation function of our ELM classification algorithm.

Then, the fuzzy groups and the indistinguishable points at the current frequency were identified quickly by the circle model. The identification results are listed in Table 4 .

Table 4. Identification of fuzzy groups for simulation test 1 .

\begin{tabular}{cc}
\hline Fuzzy Groups & Fault Elements \\
\hline 1 & $\mathrm{R} 1$ \\
\hline 2 & $\mathrm{R} 2$ \\
\hline 3 & $\mathrm{C} 1, \mathrm{R} 6$ \\
\hline 4 & $\mathrm{C} 2, \mathrm{R} 3, \mathrm{R} 4, \mathrm{R} 5$ \\
\hline 5 & $(0,0)$ \\
\hline
\end{tabular}

As shown in Table 4, there were a total of five fuzzy groups, in which $(0,0)$ stand for the voltage values that are indistinguishable at the current frequency. Hence, this group was subjected to frequency adjustment, while the other four groups were classified. Through the identification of fuzzy groups, four groups that may affect the classification decision are screened out, reducing the influence of fuzzy groups on classification accuracy.

During feature construction, three different features were created, including the $3 \mathrm{D}$ feature $\left(x_{f_{1}}, \mathrm{y}_{f_{1}}, x_{f_{2}}\right)$ designed by our method, the uncompressed $4 \mathrm{D}$ feature $\left(x_{f_{1}}, y_{f_{1}}, x_{f_{2}}, y_{f_{2}}\right)$ and the 2D feature $\left(x_{f_{1}}, y_{f_{1}}\right)$ before frequency adjustment. The three types of features were all normalized and compared with the original 3D feature. The training and test accuracies of the three normalized features and the original 3D feature are compared in Table 5 below.

Table 5. Comparison between different feature engineering approaches.

\begin{tabular}{ccc}
\hline Feature Engineering & Training Accuracy & Test Accuracy \\
\hline Normalized 3D feature & 94.7 & 93.8 \\
\hline Normalized 4D feature & 94.5 & 93.2 \\
\hline Original 3D feature & 93.9 & 92.8 \\
\hline Normalized 2D feature & 90.1 & 88.4 \\
\hline
\end{tabular}

As shown in Table 4, normalized 3D feature and normalized 4D feature had comparable accuracies, both of which are higher than those of the original 3D feature. This means the normalization method in our feature engineering can effectively improve the diagnosis accuracy. In addition, the 2D feature, which had not undergone frequency adjustment, were much poorer than the original 3D feature, which was designed by our feature engineering approach, in test accuracy. The comparison shows the frequency adjustment can make features that are indistinguishable in 2D space distinguishable in 3D space. The addition of the 3D feature can effectively enhance the test accuracy, proving the validity of our feature engineering method. 


\subsection{Simulation Test 2}

To verify its superiority, the proposed fault diagnosis algorithm was compared with the methods of Long, B. et al. [7] and Long, T. et al. [8] through a simulation test on the same circuits. The comparison mainly focuses on the test accuracy and test time.

Without loss of generality, the circuit in Figure 2 and that in Figure 8 were selected for simulation test 2 . The tolerances of the elements fall within $[-10 \%, 10 \%]$. The fault states of elements fall outside the tolerance interval: $[-50 \%,-10 \%] \mathrm{U}[10 \%, 50 \%]$. If both soft and hard faults are considered, the fault states of elements fall within $[-\infty,-10 \%] \mathrm{U}[10 \%,+\infty]$, where $-\infty$ and $+\infty$ are $X \times 10^{-4}$ and $X \times 10^{4}$, respectively ( $\mathrm{X}$ is the parameter value of an element).

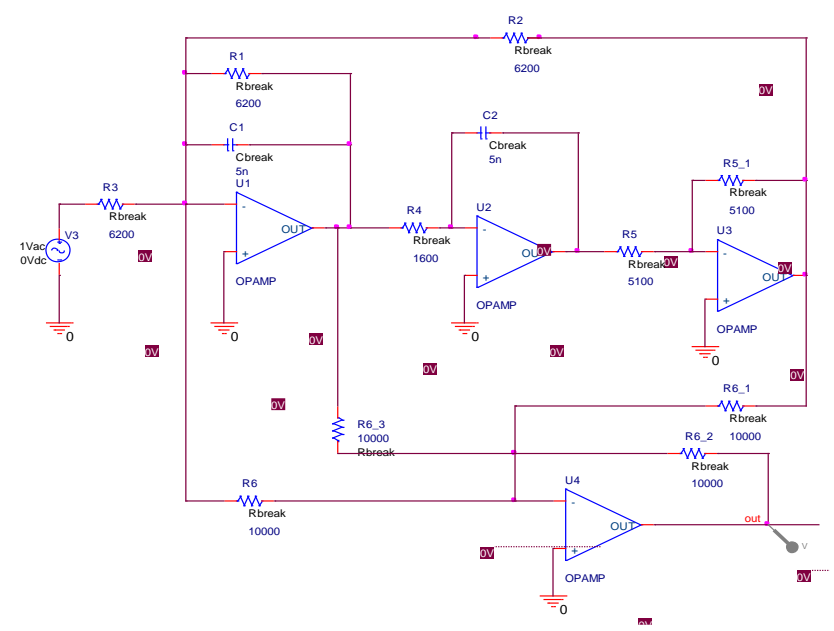

Figure 8. Sketch map of biquad quad-op amp low-pass filter.

Our method, the method of Long, B. et al. [7], and that of Long, T. et al. [8] were tested in turn. The test accuracy was measured by the false positive rate. The verification results are shown in Tables 6 and 7.

Table 6. Training and test accuracies of soft fault of Leapfrog circuit.

\begin{tabular}{cccccc}
\hline Method & $\begin{array}{c}\text { Considering Fuzzy } \\
\text { Groups? (Y/N) }\end{array}$ & $\begin{array}{c}\text { Training } \\
\text { Accuracy (\%) }\end{array}$ & $\begin{array}{c}\text { Training } \\
\text { Time (s) }\end{array}$ & $\begin{array}{c}\text { Test } \\
\text { Accuracy (\%) }\end{array}$ & Test Time (s) \\
\hline Long, B.'s method & $\mathrm{N}$ & 78.9 & 12.5 & 76.5 & 2.4 \\
\hline Long, T.'s method & $\mathrm{N}$ & 73.2 & 4.2 & 72.3 & 2.2 \\
\hline Long, B.'s method & $\mathrm{Y}$ & 92.1 & 11.2 & 90.7 & 2.2 \\
\hline Long, T.'s method & $\mathrm{Y}$ & 88.3 & 4.1 & 87.4 & 2.1 \\
\hline Our method & $\mathrm{Y}$ & 91.5 & 1.7 & 89.4 & 0.5 \\
\hline
\end{tabular}

Table 7. Training and test accuracies of soft and hard faults in two circuits.

\begin{tabular}{cccccc}
\hline Method & Type of Circuit & $\begin{array}{c}\text { Training } \\
\text { Accuracy (\%) }\end{array}$ & $\begin{array}{c}\text { Training } \\
\text { Time (s) }\end{array}$ & $\begin{array}{c}\text { Test } \\
\text { Accuracy (\%) }\end{array}$ & Test Time (s) \\
\hline Long, B.'s method & Leapfrog & 89.2 & 14.2 & 87.6 & 2.8 \\
\hline Long, T.'s method & Leapfrog & 84.6 & 5.3 & 82.3 & 2.0 \\
\hline Our method & Leapfrog & 90.3 & 2.2 & 88.8 & 0.7 \\
\hline Long, B.'s method & Biquad & 91.6 & 12.8 & 89.4 & 2.3 \\
\hline Long, T.'s method & Biquad & 87.3 & 4.9 & 85.8 & 2.1 \\
\hline Our method & Biquad & 92.2 & 1.8 & 90.1 & 0.6 \\
\hline
\end{tabular}


As shown in Table 6, Long, B.'s method and Long, T.'s method exhibited low training and test accuracies, because neither of them considers fuzzy groups. By contrast, our method reclassifies faults based on fuzzy groups, and thus achieved much better accuracy in fault diagnosis.

As shown in Table 7, when both soft and hard faults were considered, our method achieved a relatively high classification accuracy, thanks to the circle model-based feature engineering that ensures the even distribution of faults across the circuit, in the light of the circuit features. On the contrary, Long, B.'s method only considers the degree of distinction between features through feature selection, and thus achieved poorer accuracy than our method. The lowest accuracy and efficiency were observed in Long, T.'s method, which resorts to traditional measures for feature engineering and classifier design. The high efficiency of our method in fault diagnosis is attributable to the ELM classifier, which enjoys a fast classification without involving any optimization algorithm.

\section{Conclusions}

This paper proposes a novel fault diagnosis method for linear analog circuit that combines the circle model-based feature engineering with ELM. In linear analog circuits, we find that the real part $U_{o r}$ and imaginary part $U_{o j}$ of the output voltage satisfy the circle equation $\left(\mathrm{U}_{\mathrm{or}}-a\right)^{2}+\left(U_{\mathrm{oj}}-b\right)^{2}=$ $r^{2}$.The parameters of $\mathrm{a}, \mathrm{b}$ and $\mathrm{r}$ are independent from the parameter value of the component being modeled. It means that no matter how great the parameter shifting is, circle equation is always tenable. Hence, the circle equation characterizes all soft and hard faults. in detail. The circle equation can be confirmed by using three points; hence, only three simulations are needed to determine the three circle parameters $\mathrm{a}, \mathrm{b}$ and $\mathrm{r}$. Therefore, the time and space complexity are very low. Additionally, only one test node, usually the output node, is needed to model the component. Hence, it is especially applicable to the circuit under test (CUT), which has no inner accessible test nodes. The ELM classification algorithm has low computational complexity and simpler process, so it can make the classification and location of circuit fault faster. If additional test nodes are available, the fault resolution will be further enhanced.

This method has the following main advantages:

(1) Easier to model and lower space complexity. Circle modeling can be realized by simulating three sampling points at one time;

(2) Higher completeness. All fault parameters (soft fault and hard fault) are characterized in a unified way.

(3) Higher fault accuracy.

(4) Less time cost

Author Contributions: B.W. and C.S. proposed the methodology and paper organization; S.G. and J.Z. carried out the simulations and analyzed the experimental data; K.X. prepared the original draft and supervised the project; H.L. validated the obtained results; Y.Y. revised and edited the original manuscript. All authors have read and agreed to the published version of the manuscript.

Funding: This research was funded by Natural Science Foundation of Shandong Province (China) under grant number ZR2018MEE022 and Key Research and Development Program of Shandong Province (China) under grant number 2019GHY112072 and 2019GHY112051.

Acknowledgments: The authors would like to thank editors and anonymous reviewers who made constructive comments. This work was supported in part by the Natural Science Foundation of Shandong Province, China (Grant No. ZR2018MEE022) and Key Research and Development Program of Shandong Province, China (Grant No. 2019GHY112072 \& 2019GHY112051).

Conflicts of Interest: The authors declare no conflict of interest.

\section{References}

1. Bandler, J.W.; Salama, A.E. Fault diagnosis of analog circuits. Proc. IEEE 1985, 73, 1279-1325. [CrossRef]

2. Yang, C.L.; Tian, S.L.; Long, B.; Chen, F. Methods of handling the tolerance and test-point selection problem for analog-circuit fault diagnosis. IEEE Trans. Instrum. Meas. 2011, 60, 176-185. [CrossRef] 
3. Wang, P.; Yang, S. A soft fault dictionary method for analog circuit diagnosis based on slope fault mode. Control. Autom. 2006, 22, 1-23.

4. Hu, M.; Wang, H.; Hu, G.; Yang, S.Y. Soft fault diagnosis for analog circuits based on slope fault feature and BP neural networks. Tsinghua Sci. Technol. 2007, 12, 26-31. [CrossRef]

5. Fedi, G.; Giomi, R.; Luchetta, A.; Manetti, S.; Piccirilli, M.C. On the application of symbolic techniques to the multiple fault location in low testability analog circuits. IEEE Trans. Circuits Syst. II Analog Digit. Signal. Process. 1998, 45, 1383-1388. [CrossRef]

6. Tian, S.L.; Yang, C.L.; Chen, F.; Liu, Z. Circle Equation-Based Fault Modeling Method for Linear Analog Circuits. IEEE Trans. Instrum. Meas. 2014, 63, 2145-2159. [CrossRef]

7. Long, B.; Xian, W.M.; Li, M.; Wang, H.J. Improved diagnostics for the incipient faults in analog circuits using LSSVM based on PSO algorithm with Mahalanobis distance. Neurocomputing 2014, 133, 237-248. [CrossRef]

8. Long, T.; Wang, H.J.; Long, B. Test generation algorithm for analog systems based on support vector machine. Signal Image Video Process. 2011, 5, 527-533. [CrossRef]

9. Yang, C.L.; Yang, J.; Liu, Z.; Tian, S.L. Complex field fault modeling-based optimal frequency selection in linear analog circuit fault diagnosis. IEEE Trans. Instrum. Meas. 2014, 63, 813-825. [CrossRef]

10. Gao, Y.; Yang, C.L. Complex fault modeling based analog-circuit fault diagnosis. J. Univ. Electron. Sci. Technol. China 2017, 46, 540-546.

11. Villmann, T.; Kaden, M.; Hermann, W.; Biehl, M. Learning vector quantization classifiers for ROC-optimization. Comput. Stat. 2018, 33, 1173-1194. [CrossRef]

12. Gorur, K.; Bozkurt, M.R.; Bascil, M.S.; Temurtas, F. GKP signal processing using deep CNN and SVM for tongue-machine interface. Traitement Signal 2019, 36, 319-329. [CrossRef]

13. Moezzi, S.; Jalali, M.; Forghani, Y. TWSVC+: Improved twin support vector machine-based clustering. Ing. Des. Syst. D'inf. 2019, 24, 463-471. [CrossRef]

14. Meng, W.L.; Mao, C.Z.; Zhang, J.; Wen, J.; Wu, D.H. A fast recognition algorithm of online social network images based on deep learning. Traitement Signal 2019, 36, 575-580. [CrossRef]

15. Zhou, J.Y.; Tian, S.L.; Yang, C.L.; Ren, X.L. Test generation algorithm for fault detection of analog circuits based on extreme learning machine. Comput. Intell. Neurosci. 2014, 1-11. [CrossRef]

16. Zhou, J.Y.; Tian, S.L.; Yang, C.L. A novel prediction method about single components of analog circuits field modeling. Sci. World J. 2014, 2014, 1-12. [CrossRef]

17. Gao, Y.; Yang, C.L.; Tian, S.L. Phasor Analysis Based Fault Modeling and Fault Diagnosis Methods for Linear Analog Circuits. Adv. Mater. Res. 2014, 981, 3-10. [CrossRef]

18. Kumar, A.; Singh, A.P. Fuzzy classifier for fault diagnosis in analog electronic circuits. ISA Trans. 2013, 52, 816-824. [CrossRef]

19. Huang, G.B.; Zhu, Q.Y.; Siew, C.K. Extreme learning machine: Theory and applications. Neurocomputing 2006, 70, 489-501. [CrossRef]

20. Huang, G.B.; Ding, X.; Zhou, H. Optimization method based extreme learning machine for classification. Neurocomputing 2010, 74, 155-163. [CrossRef]

21. Huang, G.B.; Zhou, H.; Ding, X.; Zhang, R. Extreme learning machine for regression and multiclass classification. IEEE Tran. Sys. Man Cybern. Part B Cybern. 2012, 42, 513-529. [CrossRef] [PubMed]

22. Yu, W.X.; Sui, Y.B.; Wang, J.N. The Faults Diagnostic Analysis for Analog Circuit Based on FA-TM-ELM. J. Electron. Test. 2016, 32, 459-465. [CrossRef]

23. Shanthi, M.; Bhuvaneswari, M.C. Fault Detection in State Variable Filter Circuit Using Kernel Extreme Learning Machine (KELM) Algorithm. Microelectron. Electron. Compon. Mater. 2016, 46, 209-218.

24. Gan, X.S.; Qu, H.; Meng, X.W. Researchon ELM Soft Fault Diagnosis of Analog Circuit Based on KSLPP Feature Extraction. IEEE Access 2019, 7, 92517-92527.

(C) 2020 by the authors. Licensee MDPI, Basel, Switzerland. This article is an open access article distributed under the terms and conditions of the Creative Commons Attribution (CC BY) license (http://creativecommons.org/licenses/by/4.0/). 